\title{
The effect of timing of oseltamivir chemoprophylaxis in controlling influenza B outbreaks in long-term care facilities in Manitoba, Canada, 2017-2018: A retrospective cohort study
}

\author{
Davinder Singh, MD, MSc, JD Student; ${ }^{1}$ Depeng Jiang, PhD, Associate Professor; ${ }^{2}$ \\ Paul Van Caeseele, MD, Professor; ${ }^{3}$ Carla Loeppky, PhD, Assistant Professor ${ }^{2}$ \\ ${ }^{1}$ Faculty of Law, University of Manitoba, Winnipeg, Canada \\ ${ }^{2}$ Department of Community Health Sciences, University of Manitoba, Winnipeg, Canada \\ ${ }^{3}$ Department of Medical Microbiology, Department of Pediatrics and Child Health, University of Manitoba, Winnipeg, Canada
}

Corresponding author:

Dr. Davinder Singh, University of Manitoba, S113 - 750 Bannatyne Avenue, Winnipeg, MB Canada R3E OW3

Tel: 204-990-2072 | Fax: 204-789-3905 | davinder.singh@umanitoba.ca

\section{ABSTRACT}

A retrospective cohort study $(\mathrm{n}=8)$ was used to examine the effect of the timing of administration of oseltamivir chemoprophylaxis for the control of influenza B outbreaks among residents in long-term care facilities in Manitoba, Canada during the 2017-2018 influenza season. Delay of oseltamivir chemoprophylaxis was associated with increased odds of influenza-like illness in both univariate and multivariable analyses with an adjusted odds ratio of 1.34 (95\% Cl: 1.12-1.60) per day for influenza B.

\section{KEYWORDS}

Influenza; Outbreak; Long-term care; Oseltamivir; Prophylaxis; Public Health

\section{BACKGROUND}

In long-term care (LTC) influenza outbreaks in Manitoba, symptomatic residents receive five days of oral oseltamivir at the therapeutic dose, and all other residents receive 10 days of oseltamivir chemoprophylaxis at the prophylactic dose [1]. This approach is described in many studies, used in other countries, and is similar to the recommendations of the Infectious Diseases Society of America [1-5].

Delayed oseltamivir chemoprophylaxis is associated with increased odds of resident infection during influenza A H3N2 outbreaks in LTC facilities [6], but this has not been studied for influenza B outbreaks. Since oseltamivir is not as effective at treating influenza B as it is for influenza $A$, the effect of timing of oseltamivir chemoprophylaxis may be different [7]. This study examines the effect of the timing of administration of oseltamivir chemoprophylaxis for the control of influenza B outbreaks among residents in LTC facilities in Manitoba, Canada, controlling for other institutional factors.

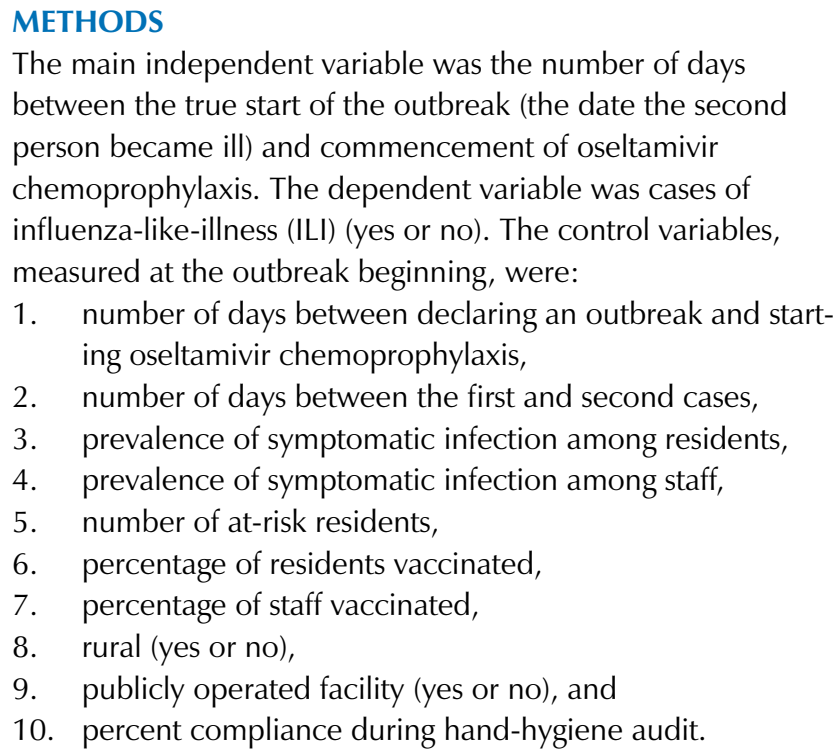
between the true start of the outbreak (the date the second person became ill) and commencement of oseltamivir chemoprophylaxis. The dependent variable was cases of influenza-like-illness (ILI) (yes or no). The control variables, measured at the outbreak beginning, were:

1. number of days between declaring an outbreak and starting oseltamivir chemoprophylaxis,

2. number of days between the first and second cases,

3. prevalence of symptomatic infection among residents,

4. prevalence of symptomatic infection among staff,

5. number of at-risk residents,

6. percentage of residents vaccinated,

7. percentage of staff vaccinated,

8. rural (yes or no),

9. publicly operated facility (yes or no), and

10. percent compliance during hand-hygiene audit. 
Outbreaks were included for analysis if:

1. they occurred between October 2017 and May 2018, and;

2. influenza type was determined.

Outbreaks were excluded if the dependent variable or the main independent variable could not be determined, or if another virus, in addition to influenza B, was detected among residents with ILI at the time of the outbreak.

The data were analyzed using a multilevel logistic regression model. All analyses were two-tailed and conducted at an alpha level (a) of 0.05 .

Additional details about methods were previously published when examining influenza A H3N2 outbreaks [6].

\section{RESULTS}

There were 20 influenza B outbreaks in LTC facilities during the 2017-2018 influenza season. Twelve outbreaks were excluded: five contained the co-detection of respiratory syncytial virus or human coronavirus, three did not report when oseltamivir was started; and four started oseltamivir on different days in different sections of the institution. The characteristics of the eight remaining influenza outbreaks can be seen in Table 1 .

Using a univariate analysis, four independent variables were statistically significant (Table 2 ): the number of days from the second case to starting oseltamivir $(t=2.93, d f=6, p=0.026)$, the number of days from declaring an outbreak to starting oseltamivir ( $t=3.48, d f=6, p=0.013$ ), the number of residents at risk $(t=3.60, d f=6, p=0.011)$, and rural location $(t=2.59$, $d f=6, p=0.041$ ).
Using a stepwise forward-modelling strategy, one variable was found to be statistically significant (Table 2): the number of days from the second case to starting oseltamivir $(t=4.18$, $\mathrm{df}=5, \mathrm{p}=0.0087$ ). The number of days from the first case to the second case $(t=2.08, d f=5, p=0.092)$, and the number of residents at risk $(t=2.31, d f=5, p=0.068)$ both trended towards significance in a two-variable model, but the number of days between the first two cases explained more variation in the sample and was included in the final model. The main effects model was assessed for co-linearity and statistically significant interactions; none were found.

The odds ratio of developing ILI for the number of days from the second case to the start of oseltamivir in the final model is 1.34 (95\% Cl: $1.12-1.60)$. This means that for every day that passes from the second case to the initiation of oseltamivir, the odds of a resident at risk of infection in the facility developing ILI increases by $34 \%$.

\section{DISCUSSION}

These data indicate that the sooner oseltamivir chemoprophylaxis is initiated, the lower the odds of secondary infection with influenza during influenza B outbreaks in LTC facilities in Manitoba. This is the first study to provide evidence supporting the rapid detection of influenza B outbreaks, and the rapid administration of oseltamivir chemoprophylaxis in an LTC resident population. Delays in this process can occur at many key points including: early recognition of illness, collection of nasopharyngeal specimens, transport of specimens to the

\begin{tabular}{|c|c|c|c|c|c|c|c|c|c|c|c|c|c|}
\hline $\begin{array}{l}\text { \# of } \\
\text { Resid }\end{array}$ & $\begin{array}{c}\text { Primary } \\
\text { Cases }^{1}\end{array}$ & $\begin{array}{c}\text { Secondary } \\
\text { Cases }\end{array}$ & $\begin{array}{c}20 \text { attack } \\
\text { rate }(\%)\end{array}$ & $\begin{array}{c}\text { Days till } \\
\text { prophylaxis² }^{2}\end{array}$ & $\begin{array}{c}\text { Days } \\
1-2^{3}\end{array}$ & $\begin{array}{c}\text { Days to } \\
\mathrm{OB}^{4}\end{array}$ & $\begin{array}{l}\text { Prev } \\
\text { Resid ILI } \\
(\%)^{5}\end{array}$ & $\begin{array}{c}\text { Prev Staff } \\
\text { ILI }(\%)^{5}\end{array}$ & $\begin{array}{l}\% \text { Staff } \\
\text { Vacc }^{5}\end{array}$ & $\begin{array}{l}\% \text { Resid } \\
\text { Vacc }^{5}\end{array}$ & $\begin{array}{l}\text { Hygiene } \\
\text { Score }^{6}\end{array}$ & $\begin{array}{l}\text { Rural } \\
(\mathrm{Y} / \mathrm{N})^{7}\end{array}$ & $\begin{array}{l}\text { Private } \\
(\mathrm{Y} / \mathrm{N})^{8}\end{array}$ \\
\hline 30 & 2 & 6 & 21 & 13 & 1 & 6 & 7 & $\mathrm{~N} / \mathrm{A}$ & 24 & 96.5 & 68 & $\mathrm{Y}$ & $\mathrm{N}$ \\
\hline 30 & 2 & 6 & 21 & 5 & 5 & 2 & 7 & 0 & $\mathrm{~N} / \mathrm{A}$ & 85 & $\mathrm{~N} / \mathrm{A}$ & $Y$ & $\mathrm{~N}$ \\
\hline 26 & 2 & 4 & 17 & 11 & 0 & 5 & 8 & 4 & 27 & 81 & 97 & Y & $\mathrm{N}$ \\
\hline 40 & 3 & 1 & 3 & 8 & 3 & 6 & 8 & $\mathrm{~N} / \mathrm{A}$ & $\mathrm{N} / \mathrm{A}$ & 74 & $\mathrm{~N} / \mathrm{A}$ & Y & $\mathrm{N}$ \\
\hline 200 & 4 & 2 & 1 & 1 & 3 & 1 & 2 & 0 & 91 & 86 & 71 & $\mathrm{~N}$ & $Y$ \\
\hline 299 & 2 & 4 & 1 & 4 & 0 & 4 & 1 & 0 & $\mathrm{~N} / \mathrm{A}$ & 89 & 40 & $\mathrm{~N}$ & $\mathrm{~N}$ \\
\hline 20 & 3 & 0 & 0 & 1 & 2 & 0 & 15 & $\mathrm{~N} / \mathrm{A}$ & $\mathrm{N} / \mathrm{A}$ & 75 & $\mathrm{~N} / \mathrm{A}$ & Y & $\mathrm{N}$ \\
\hline
\end{tabular}

Note: Resid = residents; $\mathrm{OB}=$ outbreak; Prev = prevalence; Vacc = vaccinated; N/A = not available; ILI = Influenza-like-illness; ILI is characterized as acute onset of respiratory illness with fever and cough and with one or more of the following: sore throat, arthralgia, myalgia, or prostration that could be due to influenza[1].

1 Primary cases are defined as cases of ILI occurring on or before the day that the second case occurred.

Number of days from second case to start of oseltamivir.

3 Number of days between case one and case two of the primary cases.

4 Number of days from second case to declaration of an outbreak.

5 At the start of the outbreak

Hand hygiene score in the facility during the 2017-2018 influenza season.

Rural $=$ a population less than 10,000 in the 2016 Health Canada Census $(1=$ Yes, $0=$ No $)$

8 Facilities not directly operated by the Regional Health Authority $(1=$ Yes, $0=$ No) 
laboratory, identification of viruses present, communication of results, making the decision to administer oseltamivir chemoprophylaxis, and the actual administration of oseltamivir. Rural LTC facilities experienced longer delays to initiation of oseltamivir, explaining why this variable was statistically significant with univariate analysis, but no longer significant after controlling for the time to initiation of chemoprophylaxis (Table 2). This delay could be caused by increased time to transport samples to the laboratory, and transport oseltamivir from the drug warehouse to the LTC facility in rural Manitoba. Each point of possible delay is an opportunity for a quality improvement analysis to determine if times can be reduced.

Strengths: First, Manitoba employs a common provincial approach to oseltamivir prophylaxis. Second, this study examines secondary attack rate, a more accurate approach than total attack rate. Third, oseltamivir resistance is likely not a confounder since none of the 60 influenza B samples tested in Manitoba for oseltamivir resistance were positive [8]. As well, only one of the 706 influenza B samples tested in Canada for oseltamivir resistance was positive [8]. Fourth, a multilevel model was used, accounting for both the number of outbreaks and the size of the facilities involved.

Limitations: First, the final sample size was small, increasing the likelihood that type 2 errors could be made. This also limits the generalizability of the findings since the facilities included in the analysis may not accurately represent the wider population of LTC facilities. Second, not all cases of ILI received a nasopharyngeal swab. Therefore, some cases of ILI that developed during the outbreaks may have been caused by other respiratory viruses. However, this lack of specificity

TABLE 2: Univariate and final model predictor Odds Ratios for Influenza-like-illness

\begin{tabular}{|c|c|c|}
\hline \multirow{2}{*}{$\begin{array}{l}\text { Independent Variable } \\
\text { ( } \mathrm{n}=\text { number of facilities with available information) }\end{array}$} & \multicolumn{2}{|c|}{ Model Predictions for Influenza Infection } \\
\hline & Unadjusted OR $(95 \% \mathrm{Cl})$ & Adjusted OR $(95 \% \mathrm{CI})^{1}$ \\
\hline \# Days from 2nd Case of ILI to chemoprophylaxis $(n=8)$ & $\begin{array}{c}1.29 \\
(1.04-1.59) \\
\end{array}$ & $\begin{array}{c}1.34 \\
(1.12-1.60) \\
\end{array}$ \\
\hline \# Days between 1st and 2nd Cases $(n=8)$ & $\begin{array}{c}1.07 \\
(0.52-2.18)\end{array}$ & $\begin{array}{c}1.40 \\
(0.92-2.11)\end{array}$ \\
\hline \# Days from Declaring Outbreak to Chemoprophylaxis $(n=8)$ & $\begin{array}{c}1.46 \\
(1.12-1.90) \\
\end{array}$ & - \\
\hline Prevalence of ILI among Residents ${ }^{2}(n=8)$ & $\begin{array}{c}1.08 \\
(0.82-1.44) \\
\end{array}$ & - \\
\hline \# Residents at Risk ${ }^{2}(n=8)$ & $\begin{array}{c}0.99 \\
(0.98-0.99)\end{array}$ & - \\
\hline Prevalence of ILI among Staff ${ }^{2}(n=5)$ & $\begin{array}{c}1.48 \\
(0.51-4.28) \\
\end{array}$ & - \\
\hline$\%$ Staff Vaccinated ${ }^{2}(n=4)$ & $\begin{array}{c}0.95 \\
(0.90-1.01) \\
\end{array}$ & - \\
\hline$\%$ Residents Vaccinated ${ }^{2}(n=8)$ & $\begin{array}{c}1.08 \\
(0.89-1.31) \\
\end{array}$ & - \\
\hline Rural $^{3}$ (Yes or No) $(n=8)$ & $\begin{array}{c}5.58 \\
(1.10-28.30) \\
\end{array}$ & - \\
\hline Hand Hygiene Compliance ${ }^{4}(n=5)$ & $\begin{array}{c}1.03 \\
(0.95-1.11) \\
\end{array}$ & - \\
\hline Privately $\operatorname{Run}^{5}$ (Yes or No) $(\mathbf{n}=\mathbf{8})$ & $\begin{array}{c}0.13 \\
(0.005-3.13)\end{array}$ & - \\
\hline
\end{tabular}

Note: $\mathrm{OR}=$ odds ratio; ILI = Influenza-like-illness; ILI is characterized as acute onset of respiratory illness with fever and cough and with one or more of the following: sore throat, arthralgia, myalgia, or prostration that could be due to influenza[1].

1 (-) indicates that this variable was not included in the final model

2 At the start of the outbreak

3 Rural $=$ a population less than 10,000 in the 2016 Health Canada census $(1=$ Yes, $0=$ No)

4 Hand hygiene score in the facility during the 2017-2018 influenza season. If more than one audit occurred during this time, scores were averaged

5 Facilities not directly operated by the Regional Health Authority $(1=\mathrm{Yes}, 0=\mathrm{No})$

Statistical test: multilevel logistic regression 
likely affected all institutions equally at random so only the magnitude of the result should be affected, not the presence of an effect. Third, though this study attempts to control for some of the discrepancy between how various facilities operate, some of these differences may not be accounted for by the control variables and may confound the results in an unpredictable way. Fourth, the analysis does not control for individual factors, such as age, co-morbidities, smoking status, or mobility, among the various LTC facility residents. Therefore, differences such as the number and types of co-morbidities and other demographic differences could be present and affect the results. Fifth, this study does not examine hospitalization or mortality. However, these variables are less sensitive measures of effectiveness.

\section{REFERENCES}

1. Government of Manitoba. Communicable Disease Management Protocol: Seasonal Influenza. Winnipeg, Manitoba, August, 2016, Retrieved from:: https://www.gov.mb.ca/health/publichealth/cdc/ protocol/influenza1.pdf.

2. Booy R, Lindley RI, Dwyer DE, Yin JK, Heron LG, Moffatt $C R$, et al. (2012). Treating and preventing influenza in aged care facilities: a cluster randomised controlled trial. PloS one. 7(10),e46509. doi:10.1371/journal.pone.0046509

3. Gorisek Miksic N, Ursic T, Simonovic Z, Lusa L, Lobnik Rojko P, Petrovec M, et al. (2015). Oseltamivir prophylaxis in controlling influenza outbreak in nursing homes: a comparison between three different approaches. Infection. 43(1),73-81. doi:10.1007/ s15010-014-0703-4
4. van der Sande MA, Meijer A, Sen-Kerpiclik F, Enserink R, Cools HJ, Overduin P, et al. (2014). Effectiveness of post-exposition prophylaxis with oseltamivir in nursing homes: a randomised controlled trial over four seasons. Emerg Themes Epidemiol.

11,13. doi:10.1186/1742-7622-11-13

5. Uyeki TM, Bernstein HH, Bradley JS, Englund JA, File TM, Fry AM, et al. (2019). Clinical Practice Guidelines by the Infectious Diseases Society of America: 2018 Update on Diagnosis, Treatment, Chemoprophylaxis, and Institutional Outbreak Management of Seasonal Influenza. Clin Infect Dis. 68(6),895902. doi:10.1093/cid/ciy874

6. Singh D, Jiang D, Van Caeseele P, Loeppky C. (2018). The effect of timing of oseltamivir chemoprophylaxis in controlling influenza A H3N2 outbreaks in long-term care facilities in Manitoba, Canada, 2014-2015: a retrospective cohort study. Infect Control Hosp Epidemiol. 39(8),955-60. doi:10.1017/ice.2018.115

7. Samson M, Pizzorno A, Abed Y, Boivin G. (2013). Influenza virus resistance to neuraminidase inhibitors. Antiviral Res. 98(2),17485. doi:10.1016/j.antiviral.2013.03.014

8. Government of Manitoba. Influenza Surveillance Weekly Report: Week 16. Winnipeg, Manitoba May 4, 2018, Retrieved from: https://www.gov.mb.ca/health/publichealth/surveillance/influenza/ docs/180428.pdf. 